\title{
AN ATOMIC LINEAR STARK SHIFT VIOLATING P BUT NOT T ARISING FROM THE ELECTROWEAK NUCLEAR ANAPOLE MOMENT
}

\author{
M. A. BOUCHIAT \\ Laboratoire Kastler Brossel ${ }^{1}$, \\ 24, rue Lhomond, F-75231 Paris Cedex 05, France \\ C. BOUCHIAT \\ Laboratoire de Physique Théorique de l'Ecole Normale Supérieure ${ }^{2}$ \\ 24, rue Lhomond, F-75231 Paris Cedex 05, France
}

\begin{abstract}
We propose a direct method of detection of the nuclear anapole moment. It is based on the existence of a linear Stark shift for alkali atoms in their ground state perturbed by a quadrupolar interaction of uniaxial symmetry around a direction $\hat{n}$ and a magnetic field. This shift is characterized by the T-even pseudoscalar $(\hat{n} \cdot \vec{B})(\hat{n} \wedge \vec{E} \cdot \vec{B}) / B^{2}$. It involves on the one hand the anisotropy of the hyperfine interaction induced by the quadrupolar interaction and, on the other, the static electric dipole moment arising from electroweak interactions inside the nucleus. The case of ground state Cs atoms trapped in a uniaxial (hcp) phase of solid ${ }^{4} \mathrm{He}$ is examined. From an explicit evaluation of both the hyperfine structure anisotropy and the static dipole deduced from recent empirical data about the Cs nuclear anapole moment, we predict the Stark shift. It is three times the experimental upper bound to be set on the T-odd Stark shift of free Cs atoms in order to improve the present limit on the electron EDM.
\end{abstract}

PACS. 11.30.Er - 21.90 +f - 67.80.Mg - 31.15.Ct

\footnotetext{
${ }^{1}$ Laboratoire de l'Université Pierre et Marie Curie et de l'Ecole Normale Supérieure, associé au CNRS (UMR 8552)

${ }^{2}$ UMR 8549: Unité Mixte du Centre National de la Recherche Scientifique et de l'École Normale Supérieure
} 


\section{Introduction}

It has been well demonstrated that parity violation in atomic transitions can be used to test electroweak theory [1]. In this way, the Standard Model has been confirmed convincingly in the domain of low energies. At present, refinements in experiments and theory allow more precise measurements to look for a breakdown of the Standard Model predictions and hence, new physics [2, 3, 4, 5]. The essential parameter extracted from atomic parity violation (PV) measurements is the weak nuclear charge $Q_{W}$. This electroweak parameter appears in the definition of the dominant electronnucleus $\mathrm{PV}$ potential induced by a $Z_{0}$ exchange:

$$
V_{p v}^{(1)}(r)=G_{F} / \sqrt{2} \cdot Q_{W} / 2 \cdot \gamma_{5} \cdot P_{V}(r)
$$

where the $Z_{0}$ couples to the nucleus as a vector particle, just as the photon does in the Coulomb interaction. In this $Z_{0}$ exchange, $Q_{W}$ plays the same role as the electric charge in the Coulomb interaction. $\gamma_{5}$ is the Dirac matrix which reduces to the electron helicity, $\vec{\sigma} \cdot \vec{p} / m_{e} c$, in the non-relativistic limit. The distribution $P_{V}(r)$ normalized to unity represents the weak charge distribution inside the nucleus. The physical quantity measured in atomic PV experiments is a transition electric

dipole moment, $E_{1}^{p v}$ between states with the same parity, like the $n S_{1 / 2} \rightarrow n^{\prime} S_{1 / 2}$ transitions. In particular the $6 S_{1 / 2} \rightarrow 7 S_{1 / 2}$ transition in cesium has been the subject of several experiments, the accuracy of which has been steadily increasing with time [6, 7, 8, 9, 10].

On top of this the PV electron-nucleus interaction involves also a nuclear spindependent contribution which can provide valuable and original information regarding Nuclear Physics. It is generated by an interaction of the current-current type with a vector coupling for the electron and an axial coupling for the nucleus. The associated $\mathrm{PV}$ potential $V_{p v}^{(2)}$ is given by the following expression:

$$
V_{p v}^{(2)}=G_{F} / \sqrt{2} \cdot A_{W} /(2 I) \cdot \vec{\alpha} \cdot \vec{I} \cdot P_{A}(r)
$$

where $\vec{\alpha}$ is the Dirac matrix associated with the electron velocity operator, $\vec{I}$ the nuclear spin and $P_{A}(r)$ a nuclear spin distribution normalized to unity. The weak axial moment of the nucleus, $A_{W}$, receives several contributions. The most obvious one comes from the weak neutral vector boson $Z_{0}$ with axial coupling to the nucleons. However, in the standard electroweak model the coupling constants involved nearly cancel accidentally. As first pointed out by Flambaum et al. [11], a sizeable contribution to $A_{W}$ is induced by the contamination of the atom by the PV interactions between the nucleons which take place inside the nucleus. The concept relevant to describe this interaction is the nuclear anapole moment [12]. In fact the interaction can be interpreted simply in terms of a chiral contribution to the nuclear spin 


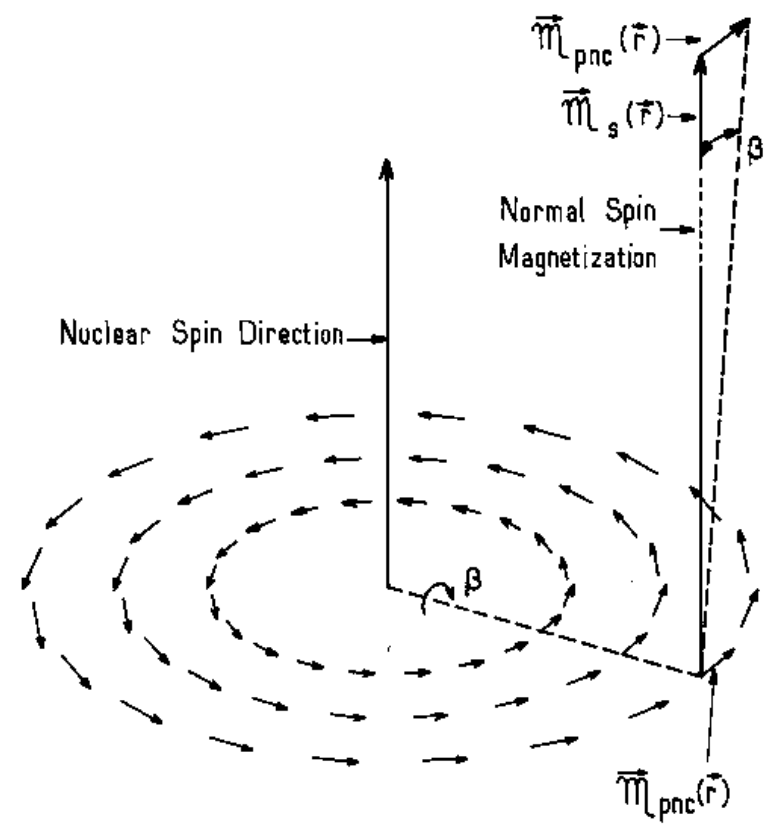

Figure 1: Simplified representation of the nuclear helimagnetism (figure adapted from C. Bouchiat [13]). The normal spin magnetization $\overrightarrow{\mathcal{M}}_{S}(\vec{r})$ is assumed to be a constant vector parallel to the nuclear spin, distributed uniformely inside a sphere. Under the influence of PV nuclear forces, the nuclear magnetization distribution inside the nucleus acquires a chiral parity non-conserving component $\overrightarrow{\mathcal{M}}_{p n c}(\vec{r})$, obtained by rotating $\overrightarrow{\mathcal{M}}_{S}(\vec{r})$ through the very small angle $\beta(r)$ around $\vec{r}$. Three chiral magnetization lines in the equatorial plane are shown. The vertical normal magnetization is actually larger than $\overrightarrow{\mathcal{M}}_{p n c}(\vec{r})$ by about six orders of magnitude. It can be shown [13] that the vertical anapole moment is given, within a constant, by the magnetic moment obtained by identifying the chiral magnetization lines with lines of electric currents.

magnetization [13, 14], as illustrated in Fig 1. In other words, one can say that the PV nuclear forces inside any stable nucleus are responsible for the nuclear anapole moment or equivalently a nuclear helimagnetism. The present paper addresses the problem of how to detect directly this unique static nuclear property characteristic of parity violation in stable nuclei.

Up to now there has been only one experimental demonstration of the nuclear anapole moment, namely that obtained very recently by the Boulder group [10]. In their experiment which gives a high precision determination of parity violation in the atomic $6 S_{1 / 2} \rightarrow 7 S_{1 / 2}$ Cs transition, this effect appears as a small relative difference, actually $\sim 5 \%$, between the $E_{1}^{p v}$ transition dipole amplitudes measured on two different hyperfine lines belonging to that same transition. In this case the dominant source of $\mathrm{P}$ without $\mathrm{T}$ violation comes from the electron-nucleon $Z_{0}$ exchange associated with the weak charge $Q_{W}$ of the nucleus. This makes the extraction of the 
nuclear spin-dependent part a most delicate matter. In view of the importance of this result for the determination of the PV pion-nucleon coupling constant, $f_{\pi}^{1}$ (see [15]), a totally independent determination is highly desirable.

It is well known that $\mathrm{T}$ reversal invariance forbids the manifestation of $V_{p v}^{(1)}$ in an atomic stationary state. However, we shall show in the following sections that in such a state $\mathrm{T}$ reversal invariance does not forbid the manifestation of $V_{p v}^{(2)}$, hence that of the nuclear helimagnetism. For a free atom, the rotation symmetry of the Hamiltonian leads to an exact cancellation of the diagonal matrix elements. This property still holds true if the rotation symmetry is broken by the application of static uniform electric and magnetic fields. However, if the symmetry is broken by the application of a static potential of quadrupolar symmetry, for instance by trapping the atoms inside a crystal of hexagonal symmetry, then, the stationary atomic states are endowed with a permanent electric dipole moment which can give rise to a linear Stark shift. This offers a novel possibility of detecting the nuclear helimagnetism having a twofold advantage:

i) In a stationary state it is the sole cause of $P$ without $T$ violation.

ii) It manifests itself by a modification of the atomic transition frequencies in an applied electric field, i.e. a linear Stark shift, providing for the first time an opportunity for demonstrating the static character of this unusual nuclear property.

There exist in the literature other proposals for a direct detection in atoms of the nuclear spin-dependent effect, i.e. without any participation from $V_{p v}^{(1)}$ :

i) One is based on the difference between the selection rules of the potentials $V_{p v}^{(1)}$ and $V_{p v}^{(2)}$. While the former acts as a scalar in the total angular momentum space and mixes only states of identical angular momentum (and opposite parity), the latter acts like a vector and mixes states of different total angular momentum. Consequently, one can find atomic transitions between states of the same parity which are allowed for the nuclear spin dependent contribution but remain forbidden for the nuclear spin independent one [16]. One such example is the $\left(6 p^{2}\right)^{3} P_{0} \rightarrow\left(6 p^{2}\right)^{1} S_{0}$ lead transition at $339.4 \mathrm{~nm}$, strictly forbidden for even isotopes, which acquires a non-vanishing matrix element $E_{1}^{p v}$ in odd isotopes owing to the PV interaction involving the nuclear spin, which mixes the $\left(6 p^{2}\right)^{1} S_{0}$ state to the $(6 p 7 s)^{3} P_{1}$ state of opposite parity [16].

ii) A second approach, invoked by several groups in the past and now under serious consideration [17], consists in the detection of an $E_{1}^{p v}$ amplitude via a right-left asymmetry appearing in hfs transition probabilities for the ground state of potassium in the presence of a strong magnetic field (magnetic and hyperfine splittings of comparable magnitude).

iii) There is also the possibility of detecting the energy difference in the NMR spectrum of enantiomer molecules 18 .

In view of the extreme difficulty of these other projects, we believe that, over 
and above its intrisic scientific interest, the linear Stark shift discussed in this paper deserves careful consideration.

The first section of this paper recalls the main angular momentum properties of the permanent nuclear spin-dependent PV electric dipole operator arising from the nuclear anapole moment. In addition we compute its magnitude for the cesium atom using recent empirical data relative to the Cs $6 S \rightarrow 7 S$ transition. The next section (sec. 2) shows that this dipole can manifest itself via a linear Stark shift only if the free atom symmetry is broken. After this we consider the case where the atom is perturbed by a crystal field of uniaxial symmetry. Here, the crystal axis $\vec{n}$, and the applied electric and magnetic fields create a chiral environment permitting the existence of a linear Stark shift, the explicit expression for which is given. In the section 3, we examine a realistic experimental situation where its observation looks reasonably feasible: this deals with Cs atoms trapped inside a ${ }^{4} \mathrm{He}$ crystal matrix of hexagonal symmetry. We have investigated quantitatively how, by breaking the atomic symmetry, the matrix induced perturbation manages to generate a linear Stark shift. Moreover, we evaluate both the matrix induced anisotropy and the shift. The details of the necessary calculation based on a semi-empirical method are given in the Appendix. In the final section we suggest another experimental approach in which the atoms are no longer submitted to a crystal field, but are instead perturbed by an intense nonresonant radiation field.

\section{The permanent nuclear spin-dependent PV electric dipole}

\subsection{Symmetry considerations}

The space-time symmetry properties of the atomic electric dipole induced by the nuclear spin dependent PV interaction have been presented before in many review papers (see for instance 19]). We recall them here for completeness, since they constitute the starting point of the linear Stark shift calculation developed in the present paper.

First, we wish to stress that the existence of the anapole moment interaction not only implies the existence of a transition dipole proportional to the nuclear spin, but also that of an electric dipole operator having diagonal matrix elements between stationary atomic states. This electric dipole is found to be proportional to the operator $\vec{s} \wedge \vec{I}$. Therefore it does not undergo the same transformation under $\mathrm{P}$ as does an ordinary dipole, since it is a pseudovector instead of a vector. We also note that it is even under T-reversal, so that the quantity $(\vec{s} \wedge \vec{I}) \cdot \vec{E}$, associated with a linear Stark shift, violates $\mathrm{P}$, but does not violate $\mathrm{T}$ invariance.

It is convenient to define $\vec{d}_{p v}\left(n^{\prime}, n\right)$ as the effective pv electric dipole moment operator acting in the tensor product $\mathcal{E}_{S} \otimes \mathcal{E}_{I}$ of the electronic and nuclear angular 
momentum spaces, which describes the transition between two $S_{1 / 2}$ subspaces corresponding to given radial quantum numbers $n$ and $n^{\prime}$. This effective dipole operator includes both contributions from potentials $V_{p v}^{(1)}$ and $V_{p v}^{(2)}$. Rotation invariance together with the fact that $V_{p v}^{(2)}$ is linear in $\vec{I}$ implies that $\vec{d}_{p v}\left(n^{\prime}, n\right)$ can be written under the following general form:

$$
\vec{d}_{p v}\left(n, n^{\prime}\right)=-i \operatorname{Im} E_{1 p v}^{(1)}\left(n, n^{\prime}\right) \vec{\sigma}+i a\left(n, n^{\prime}\right) \vec{I}+b\left(n, n^{\prime}\right) \vec{s} \wedge \vec{I}
$$

where the real quantities $a\left(n, n^{\prime}\right)$ and $b\left(n, n^{\prime}\right)$ parametrize the contribution of the nuclear spin-dependent pv potential. Time reversal invariance of $V_{p v}^{(1)}$ and $V_{p v}^{(2)}$ implies the following relations under the exchange $n \leftrightarrow n^{\prime}$ :

$$
\begin{aligned}
\operatorname{Im} E_{1 p v}^{(1)}\left(n, n^{\prime}\right) & =-\operatorname{Im} E_{1 p v}^{(1)}\left(n^{\prime}, n\right), \\
a\left(n, n^{\prime}\right) & =-a\left(n^{\prime}, n\right), \\
b\left(n, n^{\prime}\right) & =b\left(n^{\prime}, n\right) .
\end{aligned}
$$

The effective pv static dipole moment $\overrightarrow{\mathcal{D}}_{p v}=\vec{d}_{p v}(6,6)$ relative to the ground state is then given by :

$$
\overrightarrow{\mathcal{D}}_{p v}=b(6,6) \vec{s} \wedge \vec{I}=d_{I} \vec{s} \wedge \vec{I} .
$$

If we introduce the total angular momentum $\vec{F}=\vec{s}+\vec{I}$, using simple relations of angular momentum algebra, one can derive the useful identity:

$$
\vec{s} \wedge \vec{I} \equiv\left[\vec{F}^{2}, \frac{-i}{2} \vec{s}\right] .
$$

It then becomes obvious that, in low magnetic fields and without external perturbation, the dipole operator $\overrightarrow{\mathcal{D}}^{p v}$ has no diagonal matrix elements between atomic eigenstates. In fact, as demonstrated in the next section of this paper, a manifestation of this dipole requires special conditions for breaking the free-atom rotational symmetry.

\subsection{Magnitude of the permanent dipole.}

The magnitude, $d_{I}$, of the permanent dipole will play a decisive role in the assessment of the feasibility of an experiment. We are now going to perform the evaluation of $d_{I}$ in the interesting case of cesium. We proceed in two steps : first we compute directly $b(6,7)$ from experimental data, then we give a theoretical evaluation of the ratio $b(6,6) / b(6,7)$. It is convenient to use the notations of ref [0] and [13] and to rewrite $\vec{d}_{p v}(6,7)$ as:

$$
\vec{d}_{p v}(6,7)=-i \operatorname{Im} E_{1}^{p v}(6,7)\left(\vec{\sigma}+\eta \frac{\vec{I}}{I}+i \eta^{\prime} \vec{\sigma} \wedge \frac{\vec{I}}{I}\right)
$$


The nuclear spin dependent potential $V_{p v}^{(2)}$ induces a specific dependence of the pv transition dipole on the initial and final hyperfine quantum numbers, $F$ and $F^{\prime}$. In order to isolate the $V_{p v}^{(2)}$ contribution, we are led, following ref [0], to the introduction of the reduced amplitudes $d_{F F^{\prime}}$ :

$$
d_{F F^{\prime}}\left(\eta, \eta^{\prime}\right)=\frac{\left\langle 7 S, F^{\prime} M^{\prime}\left|\vec{d}_{p v}\right| 6 S, F M\right\rangle}{\left\langle F^{\prime} M^{\prime}|\vec{\sigma}| F M\right\rangle} .
$$

The amplitudes $d_{F F^{\prime}}\left(\eta, \eta^{\prime}\right)$ are tabulated in Table XXII of ref[7] and reduce to $-i \operatorname{Im} E_{1}^{p v}(6,7)$ for vanishing $\eta$ and $\eta^{\prime}$. The quantity of interest here is the ratio $r_{h f}=d_{43} / d_{34}$ which is given, to second order in $\eta$ and $\eta^{\prime}$, by :

$$
r_{h f} \simeq 1-\frac{2 I+1}{I} \eta^{\prime}
$$

Using the empirical value for the ratio $r_{h f}$ given by the last Boulder experiment [10]: $r_{h f}-1=(4.9 \pm 0.7) \times 10^{-2}$, we obtain:

$$
\eta^{\prime}=-\frac{7}{16}\left(r_{h f}-1\right)=(-2.1 \pm 0.3) \times 10^{-2} .
$$

We deduce $b(6,7)$ by a simple identification:

$$
b(6,7)=\operatorname{Im} E_{1}^{p v}(6,7) \frac{2}{I} \eta^{\prime}=(1.04 \pm 0.15 \times) 10^{-13}|e| a_{0},
$$

where we have used for $\operatorname{Im} E_{1}^{p v}(6,7)$ the empirical value obtained in ref [10]:

$$
\operatorname{Im} E_{1}^{p v}(6,7)=(-0.837 \pm 0.003) \times 10^{-11}|e| a_{0} .
$$

To compute the ratio $b(6,6) / b(6,7)$, we are going to use an approximate relation, derived in ref [13, which relates the potential $V_{p v}^{(2)}$ to $V_{p v}^{(1)}$ :

$$
V_{p v}^{(2)}(\vec{r})=K_{A} \frac{A_{W}}{Q_{W}} 2 \vec{j} \cdot \frac{\vec{I}}{I} V_{p v}^{(1)}(\vec{r}) .
$$

Here $K_{A}$ is a constant very close to unity which depends weakly upon the shape of the nuclear distributions $P_{V}(r)$ and $P_{A}(r) ; \vec{j}$ is the single electron angular momentum and since, as we shall see, only single particle states with $j=1 / 2$ are involved, we can write hereafter $2 \vec{j}=\vec{\sigma}$.

This relation, valid for high $\mathrm{Z}$ atoms like cesium, hinges upon the fact that the matrix elements $\left\langle n^{\prime} p_{3 / 2}\left|V_{p v}^{(2)}\right| n s_{1 / 2}\right\rangle$ involving $p_{3 / 2}$ states are much smaller- by a factor $2 \times 10^{-3}$ - than those which involve $p_{1 / 2}$ states, $\left\langle n^{\prime} p_{1 / 2}\left|V_{p v}^{(2)}\right| n s_{1 / 2}\right\rangle$. This is easily 
verified in the one-particle approximation since the radial wave functions at the surface of the nucleus are very close to Dirac Coulomb wave functions for an unscreened charge $Z$. It is argued in ref [13] that this property remains true, to the level of few $\%$, when $V_{p v}^{(2)}(\vec{r})$ is replaced by the non local potential $U_{p v}^{(2)}\left(\vec{r}, \vec{r}^{\prime}\right)$, which describes the core polarization effects within the R.P.A. approximation [?

The contributions of $V_{p v}^{(i)}$ to the effective dipole operator $\vec{d}_{p v}\left(n, n^{\prime}\right)$ are given as the sum of the two operators:

$$
\begin{aligned}
& \vec{A}^{(i)}=P\left(n^{\prime} S_{1 / 2}\right) V_{p v}^{(i)} G\left(E_{n^{\prime}}\right) \vec{d} P\left(n S_{1 / 2}\right), \\
& \vec{B}^{(i)}=P\left(n^{\prime} S_{1 / 2}\right) \vec{d} G\left(E_{n}\right) V_{p v}^{(i)} P\left(n S_{1 / 2}\right),
\end{aligned}
$$

where $\vec{d}$ is the electric dipole operator, $G\left(E_{n}\right)=\left(E_{n}-H_{\text {atom }}\right)^{-1}$ the Green function operator relative to the atomic hamiltonian; $P\left(n S_{1 / 2}\right)$ and $P\left(n^{\prime} S_{1 / 2}\right)$ stand for the projectors upon the subspaces associated with the configurations $n S_{1 / 2}$ and $n^{\prime} S_{1 / 2}$; $E_{n}$ and $E_{n^{\prime}}$ are the corresponding binding energies. It follows immediatly from the Wigner-Eckart theorem that the operators $\vec{A}^{(1)}$ and $\vec{B}^{(1)}$ can be written as:

$$
\vec{A}^{(1)}=i h\left(n, n^{\prime}\right) \vec{\sigma} ; \quad \vec{B}^{(1)}=i k\left(n, n^{\prime}\right) \vec{\sigma} .
$$

Using now the relation given in equation (12) and the commutation of $\vec{\sigma} \cdot \vec{I}$ with the pseudoscalar $V_{p v}^{(1)}$, one gets the following expressions for $\vec{A}^{(2)}$ and $\vec{B}^{(2)}$ :

$$
\begin{aligned}
\vec{A}^{(2)} & =i K_{A} \frac{A_{W}}{Q_{W}} h\left(n, n^{\prime}\right)\left(\vec{\sigma} \cdot \frac{\vec{I}}{I}\right) \vec{\sigma}, \\
\vec{B}^{(2)} & =i K_{A} \frac{A_{W}}{Q_{W}} k\left(n, n^{\prime}\right) \vec{\sigma}\left(\vec{\sigma} \cdot \frac{\vec{I}}{I}\right) .
\end{aligned}
$$

We arrive finally at an expression for $\vec{d}_{p v}\left(n, n^{\prime}\right)$ which can be used to compute the ratio $b(6,6) / b(6,7)$ :

$$
\begin{aligned}
\vec{d}_{p v}\left(n, n^{\prime}\right)= & i\left(\vec{\sigma}+K_{A} \frac{A_{W}}{Q_{W}} \frac{\vec{I}}{I}\right)\left(h\left(n, n^{\prime}\right)+k\left(n, n^{\prime}\right)\right)+ \\
& \vec{\sigma} \wedge \frac{\vec{I}}{I} K_{A} \frac{A_{W}}{Q_{W}}\left(h\left(n, n^{\prime}\right)-k\left(n, n^{\prime}\right)\right) .
\end{aligned}
$$

\footnotetext{
${ }^{3}$ To check the validity of the relation (12) we have compared the values for $\eta$ and $\eta^{\prime}$ obtained in this way with those deduced from a direct computation [20] of $\vec{d}_{p v}(6,7)$. The two results agree to better than $10 \%$.
} 
Time reversal invariance implies $h(n, n)=-k(n, n)$ so that we can write the sought for ratio $b(6,6) / b(6,7)$ as:

$$
\frac{b(6,6)}{b(6,7)}=\frac{2 h(6,6)}{h(6,7)-k(6,7)}
$$

The amplitudes $h(6,6), h(6,7)$ and $k(6,7)$ can be computed from the formulas given in Eqs. (13) and (14). We have used the explicit values of the radial matrix elements (parity mixing and allowed electric dipole amplitudes) for the intermediate statest $6 P_{1 / 2}-9 P_{1 / 2}$ and the energy differences involved, which are tabulated in ref. 20] (Table IV)国. We obtain in this way:

$$
b(6,6) / b(6,7)=4.152 / 1.86=2.27 .
$$

Combining the above result with the value of $b(6,7)$ given by equation (11) we obtain the following estimate for $d_{I}$ :

$$
d_{I} \simeq 2.36 \times 10^{-13}|e| a_{0}
$$

believed to be about $15 \%$ accurate.

It is of interest to compare $d_{I}$ with the P-odd T-odd EDM of the Cs atom obtained from a theoretical evaluation using the latest experimental upper bound for the electron EDM [21]:

$$
\left|d_{e}\right| \leq 7.5 \times 10^{-19}|e| a_{0}
$$

Using for the cesium anti-screening factor the theoretical value [22]: $120 \pm 10$, one gets the following upper bound for the cesium EDM, namely the experimental sensitivity to be reached for improving the existing bound on $\left|d_{e}\right|$ :

$$
\left|d_{C s E D M}\right| \leq 9.0 \times 10^{-17}|e| a_{0} .
$$

We are going to use Eqs.(5) and (19) for calculating the linear Stark shift. It is interesting to note here that these equations predict also the magnitude of the pv transition dipole involved in an eventual Cs project which would be based on the observation of hyperfine transitions in the Cs ground state, analogous to the potassium project mentioned in the introduction (see also [17]). Therefore both a project of this kind and the linear Stark shift discussed here aim at the determination of the same physical parameter, $d_{I}$, but only the observation of a dc Stark shift would prove its static character.

\footnotetext{
${ }^{4}$ We use here the fact that, as noted by several authors, most of the sum $(\approx 98 \%)$ comes from the four states $6 P_{1 / 2}, 7 P_{1 / 2}, 8 P_{1 / 2}, 9 P_{1 / 2}$.

${ }^{5}$ Note that a misprint in table IV of ref [20] has caused an interchange between the contents of columns 1 and 2 of its lower half (entitled "7S perturbed").
} 


\section{The linear Stark shift induced by $V_{p v}^{(2)}$}

\subsection{Need for breaking the rotation symmetry of the atomic Hamiltonian}

The parity conserving spin Hamiltonian in presence of a static magnetic field $\vec{B}_{0}$ is:

$$
H_{\text {spin }}=A \vec{s} \cdot \vec{I}-g_{s} \mu_{B} \vec{s} \cdot \vec{B}_{0}-\gamma_{I} \vec{I} \cdot \vec{B}_{0} .
$$

From section 2, we have seen that, to first order in the electric field, the effect of $V_{p v}^{(2)}$ in presence of an applied electric field can be described by the following Stark Hamiltonian:

$$
H_{p v}^{s t}=d_{I} \vec{s} \wedge \vec{I} \cdot \vec{E} \equiv-d_{I} \frac{i}{2}\left[\vec{F}^{2}, \vec{s} \cdot \vec{E}\right] .
$$

We have noted that the above identity implies the vanishing of the average value of $H_{p v}^{s t}$ in the low magnetic field limit. We are going to show that this null result still remains valid for arbitrary values and orientations of the magnetic field.

To do this we consider the transformation properties of both $H_{s p i n}$ and $H_{p v}^{s t}$ under the symmetry $\Theta$, defined as the product of $T$ reversal by a rotation of $\pi$ around the unit vector $\hat{u}=\vec{E} \wedge \vec{B}_{0} /\left|E B_{0}\right|$, the rotation $R(\hat{u}, \pi)$. It should be stressed that the rotation $R(\hat{u}, \pi)$ and the symmetry $\Theta$ considered here are quantum mechanical transformations acting only on the spin states. The external fields are considered as real $c$-numbers and are not affected. One sees immediately that $H_{\text {spin }}$ is invariant under the symmetry $\Theta=T R(\hat{u}, \pi)$, while $H_{p v}^{s t}$ changes sign. We conclude that, in order to suppress the linear Stark shift cancellation we have to break the $\Theta$ symmetry.

This symmetry breaking can be achieved, for instance, by perturbing the atomic $S_{1 / 2}$ state with a crystal field compatible with uniaxial symmetry along the unit vector $\vec{n}$. A practical realization looks feasible, since it has been demonstrated that Cs atoms can be trapped in a solid matrix of helium having an hexagonal symmetry [23] (see also section 3). In this case the alkali S state is perturbed by the Hamiltonian?:

$$
H_{b}(\vec{n})=\lambda_{b} \cdot\left(\frac{e^{2}}{2 a_{0}}\right)\left((\vec{\rho} \cdot \vec{n})^{2}-\frac{1}{3} \rho^{2}\right)
$$

where both $\lambda_{b}$ and $\rho=r / a_{0}$ are expressed in atomic units. The perturbed atomic state is now a mixture of $\mathrm{S}$ and $\mathrm{D}$ states, with no component of the orbital angular momentum along the $\vec{n}$ axis. The spin Hamiltonian is modified and an anisotropic hyperfine interaction is induced by the $\mathrm{D}$ state admixture. The new spin Hamiltonian reads:

$$
\widetilde{H}_{\text {spin }}=A_{\perp} \vec{s} \cdot \vec{I}+\left(A_{\|}-A_{\perp}\right)(\vec{s} \cdot \vec{n})(\vec{I} \cdot \vec{n})-g_{s} \mu_{B} \vec{s} \cdot \vec{B}_{0}-\gamma_{I} \vec{I} \cdot \overrightarrow{B_{0}} .
$$

\footnotetext{
${ }^{6}$ It has been shown 23 that in the bubble enclosing the cesium atom there is a small overlap between the cesium and the helium orbitals. As a consequence, the axially symmetric crystal potential inside the bubble can be well approximated by a regular solution of the Laplace equation.
} 
It is easily verified that, if $\vec{n}$ lies in the $(\vec{B}, \hat{u})$ plane, with non-zero components along both $\vec{B}$ and $\hat{u}$, this perturbed atomic Hamiltonian is no longer invariant under the transformation $\Theta$.

Another possible method for breaking the symmetry of $H_{\text {spin }}$ will be presented in section 4 .

\subsection{Strong magnetic field limit $\left(\gamma_{s} B_{0} \gg A_{\perp}, A_{\|}\right)$}

The anisotropy axis is defined as:

$$
\vec{n}=\cos \psi \hat{z}+\sin \psi \hat{x} .
$$

Let us consider the nuclear spin Hamiltonian associated with the restriction of $H_{\text {spin }}$ to the electronic eigenstate $\mathcal{E}\left(\tilde{n}_{s}, m_{s}\right)$ perturbed by the quadrupolar potential $H_{b}(\vec{n})$ :

$$
\begin{aligned}
H_{\left(m_{s}\right)}^{e f f} & =A_{\perp} m_{s} I_{z}+m_{s}\left(A_{\|}-A_{\perp}\right)\left(\sin \psi \cos \psi I_{x}+\cos ^{2} \psi I_{z}\right)+\gamma_{s} B_{0} m_{s}-\gamma_{I} B_{0} I_{z} \\
& =m_{s}\left[\gamma_{s} B_{0}+I_{z}\left(A_{\perp} \sin ^{2} \psi+A_{\|} \cos ^{2} \psi-\frac{\gamma_{I} B_{0}}{m_{s}}\right)+I_{x}\left(A_{\|}-A_{\perp}\right) \sin \psi \cos \psi\right]
\end{aligned}
$$

$H_{\left(m_{s}\right)}^{e f f}$ is identical to the Hamiltonian seen by an isolated nucleus coupled to an effective magnetic field, $\vec{B}^{e f f}\left(m_{s}\right)$, having the following components:

$$
\begin{aligned}
B_{x}^{e f f} & =-\left(A_{\|}-A_{\perp}\right) \sin \psi \cos \psi \frac{m_{s}}{\gamma_{I}} \\
B_{y}^{e f f} & =0 \\
B_{z}^{e f f} & =B_{0}-\left(A_{\perp} \sin ^{2} \psi+A_{\|} \cos ^{2} \psi\right) \frac{m_{s}}{\gamma_{I}},
\end{aligned}
$$

or equivalently :

$$
B_{x}^{e f f}=B^{e f f} \sin \alpha, \quad B_{y}^{e f f}=0, \quad B_{z}^{e f f}=B^{e f f} \cos \alpha,
$$

where

$$
\tan \alpha=\frac{\left(A_{\|}-A_{\perp}\right) \sin \psi \cos \psi m_{s}}{-\gamma_{I} B_{0}+\left(A_{\perp} \sin ^{2} \psi+A_{\|} \cos ^{2} \psi\right) m_{s}} .
$$

In other words, the direction $\hat{z}^{\text {eff }}$ of $\vec{B}^{e f f}$ can be deduced from the $\hat{z}$ axis by a rotation $\mathcal{R}(\hat{y}, \alpha)$ by an angle $\alpha$ around the $\hat{y}$ axis. Hence, the eigenstates of $H_{\left(m_{s}\right)}^{\text {eff }}$ are $\mid m_{s} \tilde{m}_{I}>$, where $\tilde{m}_{I}$ now stands for the $z$-component of the spin $\widetilde{\vec{I}}$ resulting from $\vec{I}$ through the rotation $\mathcal{R}(\hat{y},-\alpha)$.

$$
\widetilde{I}_{z}=\widetilde{\vec{I}} \cdot \hat{z}=\vec{I} \cdot \mathcal{R}(\hat{y}, \alpha) \hat{z}=\cos \alpha I_{z}+\sin \alpha I_{x}
$$


We can now compute the linear Stark shift associated with the Hamiltonian $H_{s t}^{p v}$ given by Eq.(22), supposing the $\vec{E}$ field directed along the $\hat{y}$ axis:

$$
\begin{aligned}
\Delta E_{s t} & =\left\langle m_{s} \tilde{m}_{I}\left|d_{I} E s_{z} I_{x}\right| m_{s} \tilde{m}_{I}\right\rangle \\
& =d_{I} E m_{s}\left\langle\tilde{m}_{I}\left|I_{x}\right| \tilde{m}_{I}\right\rangle \\
& =d_{I} E m_{s}\left\langle m_{I}|I \cdot \mathcal{R}(\hat{y}, \alpha) \hat{x}| m_{I}\right\rangle \\
& =-d_{I} E m_{s} m_{I} \sin \alpha .
\end{aligned}
$$

If we suppose $\gamma_{I} B_{0} \ll A_{\|}, A_{\perp} \ll\left|\gamma_{s}\right| B_{0}$ and $\left|A_{\|}-A_{\perp}\right| \ll A_{\|}+A_{\perp}$, we obtain:

$$
\tan \alpha \approx \frac{A_{\|}-A_{\perp}}{\frac{1}{2}\left(A_{\|}+A_{\perp}\right)} \sin \psi \cos \psi \approx \sin \alpha
$$

which yields the simplified expression:

$$
\Delta E_{s t}=-d_{I} E m_{s} m_{I} \frac{A_{\|}-A_{\perp}}{A_{\|}+A_{\perp}} \sin 2 \psi .
$$

In this approximation, $\Delta E_{s t}$ can be considered as a modification of the hyperfine constant linear in the applied electric field.

In order to show up the transformation properties of $\Delta E_{s t}$, it is useful to express this last result in terms of the two fields, $\vec{E}$ and $\vec{B}$, and the unit vector $\hat{n}$ which defines the anisotropy axis:

$$
\Delta E_{s t}=-2 d_{I} m_{s} m_{I} \frac{\left(\hat{n} \cdot \vec{B}_{0}\right)\left(\hat{n} \cdot \vec{E} \wedge \vec{B}_{0}\right)}{\vec{B}_{0}^{2}} \frac{A_{\|}-A_{\perp}}{A_{\|}+A_{\perp}}
$$

From this expression, it is clearly apparent that the linear shift breaks space reflexion symmetry but preserves time reversal invariance. It differs from the $\mathrm{P}$ and $\mathrm{T}$ violating linear Stark shift arising from an electron EDM by the fact that it cancels out when the quadrupolar anisotropy of the ground state vanishes. It is also obvious from Eq. (29) that, in the strong field limit, the size of the Stark shift depends only on the orientation of $\vec{B}$ relative to $\vec{E}$ and $\vec{n}$ and not on the strength of the magnetic field. Figure 2 represents two mirror-image configurations of the experiment.

\subsection{Limit of low magnetic fields and small anisotropy}

We now consider the limit $\left|A_{\|}-A_{\perp}\right| \ll \gamma_{s} B_{0} \ll A_{\perp}, A_{\|}$.

The linear Stark shift can be computed by using second order perturbation theory. $H_{\text {spin }}$ is perturbed by both $H_{p v}^{s t}$ and $H_{b}(\vec{n})$, the latter being responsible for the 

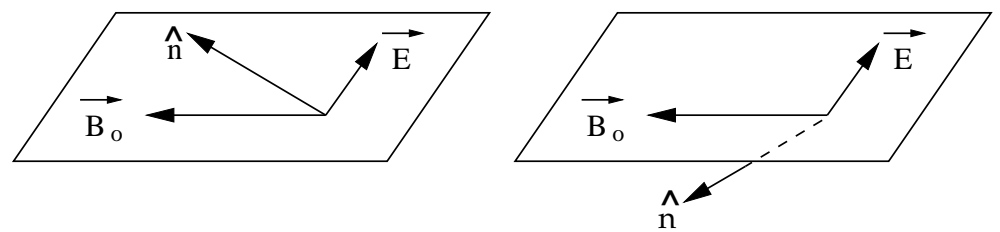

Figure 2: Two mirror-image and T-reversal symmetric experimental configurations corresponding to opposite values of the pseudoscalar $\left(\hat{n} \cdot \vec{B}_{0}\right)\left(\hat{n} \cdot \vec{E} \wedge \vec{B}_{0}\right) / \vec{B}_{0}^{2}$.

anisotropy contribution to $H_{\text {spin }}$, i.e. $\left(A_{\|}-A_{\perp}\right)(\vec{s} \cdot \hat{n})(\vec{I} \cdot \hat{n})$. The fields $\vec{B}_{0}$ and $\vec{E}$ are still taken parallel to $\hat{z}$ and $\hat{y}$ respectively. We find:

$$
\begin{gathered}
\Delta E_{s t}(F, M)=2\left(A_{\|}-A_{\perp}\right) d_{I} E \cos \psi \sin \psi \times \\
\times \sum_{F^{\prime} \neq F, M^{\prime}} \frac{\left\langle F M\left|s_{z} I_{x}+s_{x} I_{z}\right| F^{\prime} M^{\prime}\right\rangle\left\langle F^{\prime} M^{\prime}\left|s_{z} I_{x}-s_{x} I_{z}\right| F M\right\rangle}{E_{F M}-E_{F^{\prime} M^{\prime}}} .
\end{gathered}
$$

Since the operator $\vec{s} \wedge \vec{I}$ is identical to the commutator $\left[\vec{F}^{2},-\frac{i}{2} \vec{s}\right]$, we see that only the hyperfine states $F^{\prime} \neq F$ with $M^{\prime}=M \pm 1$ contribute to the sum. Therefore, in the energy denominator we can neglect the Zeeman contribution which is small compared to the hyperfine splitting and, in the sum, we can factorize out the energy denominator $2(F-I) A_{\|}\left(I+\frac{1}{2}\right)$. Since $F^{\prime}=F$ does not contribute, the resulting sum can be performed using a closure relation:

$$
\Delta E_{s t}(F, M)=\frac{\left(A_{\|}-A_{\perp}\right) d_{I} E}{2(F-I) A_{\|}\left(I+\frac{1}{2}\right)} \sin 2 \psi\left\langle F M\left|\left(s_{z} I_{x}+s_{x} I_{z}\right)\left(s_{z} I_{x}-s_{x} I_{z}\right)\right| F M\right\rangle .
$$

Using standard properties of spin 1/2 matrices, we can transform the diagonal matrix element above into $\frac{1}{4}\left\langle F M\left|\left(I_{x}^{2}-I_{z}^{2}\right)\right| F M\right\rangle$. Once taken into account the axial symmetry of the unperturbed atomic state, it still simplifies to $\frac{1}{8}\left\langle F M\left|\left(\vec{I}^{2}-3 I_{z}^{2}\right)\right| F M\right\rangle$.

We arrive at the final expression:

$$
\Delta E_{s t}(F, M)=k(F, M) \frac{A_{\|}-A_{\perp}}{A_{\|}+A_{\perp}} d_{I} E \sin 2 \psi,
$$

where

$$
k(F, M)=2(F-I)\left\langle F M\left|\frac{1}{2}\left(\vec{I}^{2}-3 I_{z}^{2}\right)\right| F M\right\rangle /(2 I+1) .
$$

The Stark shift coefficients $k(F, M)$ for ${ }_{55}^{133} \mathrm{Cs}(\mathrm{I}=7 / 2)$ are listed in Table 1.

We note that $\Delta E_{s t}$ depends on $M^{2}$ so the linear Stark shifts of the Zeeman splittings $E(F, M)-E(F, M-1)$ have opposite signs for $M>0$ and $M<0$ (see figure 3):

$$
E(4, M)-E(4, M-1)=\hbar \omega_{s} M /(2 I+1)+\Delta E_{s t}(4,|M|)-\Delta E_{s t}(4,|M-1|) .
$$



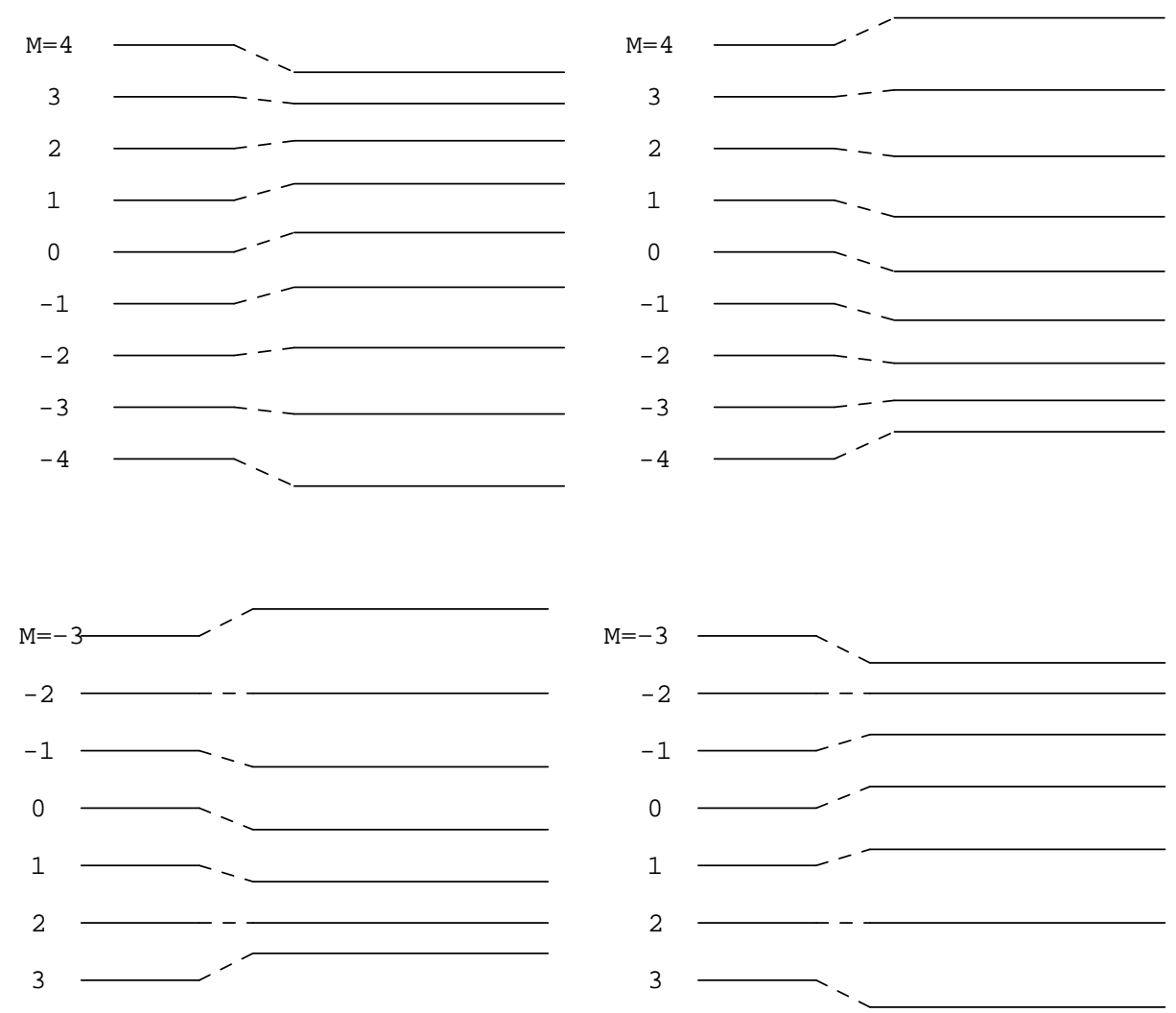

Figure 3: Energies of the different F, M states of the ground configuration of ${ }^{133} \mathrm{Cs}$ showing the linear Stark shift largely magnified. The two figures correspond to situations realizing opposite signs of the pseudoscalar $\mathcal{P}$ defined in the text (Left: $\mathcal{P}>0$; right: $\mathcal{P}<0$ ). Top: $\mathrm{F}=4$; bottom: $\mathrm{F}=3$. 


\begin{tabular}{|c|c|c|c|c|c|}
\hline$|M|$ & 0 & 1 & 2 & 3 & 4 \\
\hline $\mathrm{k}(4,|M|)$ & $15 / 16$ & $51 / 64$ & $3 / 8$ & $-21 / 64$ & $-21 / 16$ \\
\hline $\mathrm{k}(3,|M|)$ & $-15 / 16$ & $-45 / 64$ & 0 & $75 / 64$ & \\
\hline
\end{tabular}

Table 1: Linear Stark shift coefficients $\mathrm{k}(4, \mathrm{M})$ and $\mathrm{k}(3, \mathrm{M})$ of the different $\mathrm{F}, \mathrm{M}$ substates of the natural cesium ground state

As expected, once again the pseudoscalar

$$
\mathcal{P}=\frac{A_{\|}-A_{\perp}}{A_{\|}+A_{\perp}} \frac{\left(\hat{n} \cdot \vec{B}_{0}\right)\left(\hat{n} \cdot \vec{E} \wedge \vec{B}_{0}\right)}{B_{0}^{2}},
$$

plays an essential role. If $\mathcal{P}>0$, there is a contraction of the Zeeman splittings belonging to the $\mathrm{F}=4$ hyperfine state for positive values of $M$ and a dilatation for negative ones, as shown by Figure 3. The situation is reversed when the sign of $\mathcal{P}$ is changed. In the $\mathrm{F}=3$ hyperfine state, splitting contraction also occurs for $M>0$ with $\mathcal{P}>0$ and for $M<0$ with $\mathcal{P}<0$. This behavior could help to discriminate the linear Stark shift induced by the nuclear helimagnetism from spurious effects. The largest shift between two contiguous sublevels is expected to occur for the couple of states $F=3, M=|3| \rightarrow F=3, M=|2|$.

From Table 1 and Eqs. (19) and (31) we predict:

$$
\Delta E_{s t}(3,3)-\Delta E_{s t}(3,2)=\frac{75}{64} \frac{A_{\|}-A_{\perp}}{A_{\|}+A_{\perp}} \sin 2 \psi d_{I} \cdot E
$$

with $\frac{75}{64} d_{I} \simeq 2.76 \times 10^{-13}|e| a_{0}$.

As in the strong field limit, we note that the size of $\Delta E_{s t}$ depends only on the direction of $\vec{B}$.

\subsection{Analogy between this shift and the PV energy shift searched for in enantiomer molecules}

We would like to stress that from the point of view of symmetry considerations there exists a close analogy between the linear Stark shift induced by the anapole moment and the energy shift which is searched for in enantiomer molecules [24. Indeed in the present configuration the three vectors $\vec{E}, \vec{B}$ and $\vec{n}$ which are non-coplanar are sufficient to place the atom in a chiral environment similar to that experienced by an atomic nucleus inside a chiral molecule. Between two mirror-image environments an energy difference is predicted exactly like between two mirror-image molecules. 


\section{Experimental considerations and order of magnitude estimate}

We now consider an experimental situation which looks like a possible candidate for the observation of the linear Stark shift discussed in the previous sections. It has been demonstrated experimentally [25] that cesium atoms can be trapped in solid matrices of ${ }^{4} \mathrm{He}$. At low pressures, solid helium cristallizes in an isotropic body-centered cubic (bcc) phase, but also in a uniaxial hexagonal close packed (hcp) phase. Optically detected magnetic resonance has proved to be a sensitive tool for investigating the symmetry of the trapping sites. The group of A. Weis has reported the observation in the hexagonal phase of zero-field magnetic resonance spectra and magnetic dipoleforbidden transitions which they interpret in terms of a quadrupolar distorsion of the atomic bubbles [26]. Particularly relevant here is their observation of the matrixinduced lifting of the Zeeman degeneracies in zero field. This is attributed to the combined effect of two interactions, the quadrupolar interaction of the form $H_{b}(\vec{n})=$ $\lambda_{b}\left((\vec{\rho} \cdot \vec{n})^{2}-\frac{1}{3} \rho^{2}\right)$ between the cesium atom and the He matrix on the one hand, and the hyperfine interaction in the Cs atom on the other.

Provided that $\vec{F}^{2}$ is still a good quantum number, it is easily shown from general symmetry considerations, that the anisotropy of the hyperfine interaction induced by the (hcp) crystal potential can be represented, within a given hyperfine multiplet, by the effective perturbation:

$$
H_{e f f}=C_{e f f}(F) \cdot\left((\vec{F} \cdot \hat{n})^{2}-\frac{1}{3} \vec{F}^{2}\right) .
$$

The constants $C_{e f f}(F)$ can be easily related to the anisotropic hyperfine constants appearing in the spin hamiltonian $\widetilde{H}_{\text {spin }}$ introduced in Eq.(23) of the previous section:

$$
C_{e f f}(F=I \pm 1 / 2)= \pm\left(A_{\|}-A_{\perp}\right) / 8 .
$$

In a uniaxial crystal, when the atoms are optically polarized along the crystal axis in the absence of external magnetic fields, the lifting of the degeneracy between Zeeman sublevels induced by $H_{\text {eff }}$ should make it possible to drive magnetic resonance transitions between these levels. One would expect to deduce the hyperfine anisotropy from the observed spectra. At first sight, the zero-field magnetic resonance spectra observed by Weis et al. would seem to match this prediction. However, their experiment has been performed in a polycristalline (hcp) sample. The effects observed in this situation result from averaging over the distribution of the microcrystal axes. For each microcrystal, there exists a quantization axis, $\hat{z}$, which diagonalizes the hyperfine level density matrix. Immediately a question arises as to the direction of the quantization axis $\hat{z}$ with respect to the microcrystal symmetry axis $\vec{n}$. If the population differences resulted, say, from the Boltzmann factor, then $\hat{z}$ would be along $\vec{n}$, since in the zero magnetic field limit there is no other preferred direction. In such 
a situation, there would be no difference between the spectra for a polycrystal and a monocrystal. But in the experimental situation considered here, the population differences are induced by an optical pumping mechanism which provides a second preferred direction: the direction of the photon angular momentum along $\vec{k}$. The microcrystal density matrix is then expected to keep some memory of the direction of $\vec{k}$. So, two directions $\vec{n}$ and $\vec{k}$ compete in the determination of the quantization axis $\hat{z}$. To proceed further, we consider the extreme case where $\hat{z}$ is taken along $\vec{k}$, together with an assumed isotropic distribution of microcrystal axes. It is then easily seen that the lines associated with the hyperfine anisotropy $H_{\text {eff }}$ collapse into a single asymmetric line when the average is performed over the polycrystal. Clearly, one at least of the two preceeding assumptions is too drastic, most likely the isotropy of the $\vec{n}$ distribution. It is indeed likely that the optical pumping process is more efficient for microcrystals having a preferred orientation with respect to the photon angular momentum. Such a selection mechanism would then lead to an effective anisotropic distribution of $\vec{n}$, and in this way a spectrum of separated lines can be recovered. From the above qualitative considerations, it clearly follows that the final interpretation of the the zero-field resonances requires a detailed analysis of the optical pumping process for Cs atoms trapped inside deformed bubbles of arbitrary orientation. The corresponding theoretical investigation is currently underway in A. Weis's group.

Meanwhile, to plan any experiment, we still need to know about the physical origin and the magnitude of the ratio $\frac{A_{\|}-A_{\perp}}{A_{\|}+A_{\perp}}$, which governs the magnitude of the electroweak linear Stark shift. We are going to present now the result of an investigation which has led us both to a physical understanding and a reasonably accurate estimate of the sought after parameter. We have chosen to devote an appendix to a detailed description of our semi-empirical approach, which consists in relating the hyperfine anisotropy to another measured physical quantity. Here we shall give a brief summary of our procedure and present the final result.

We start from the remark that there really does exist a mechanism able to generate an hyperfine anisotropy to first order in the "bubble" Hamiltonian $H_{b}(\vec{n})$. The $n D_{3 / 2}$ state is indeed mixed to the $6 S_{1 / 2}$ state under the effect of $H_{b}(\vec{n})$, and we note then that the hyperfine interaction has non-zero off-diagonal matrix elements between $S_{1 / 2}$ and $D_{3 / 2}$ states. In fact, it has been shown previously [27] that the $\left\langle n S_{1 / 2}\left|H_{h f}\right| n^{\prime} D_{3 / 2}\right\rangle$ matrix elements are not easy to calculate, because they are dominated by the contribution coming from many-body effects, due to the existence of an approximate selection rule which suppresses the single particle matrix element. However, as we show in the appendix, the variation of the matrix elements $\left\langle n^{\prime} S_{1 / 2}\left|H_{h f}\right| n^{\prime \prime} D_{3 / 2}\right\rangle$ with respect to the binding energies $\mathcal{E}_{n^{\prime} S_{1 / 2}}$ and $\mathcal{E}_{n^{\prime \prime} D_{3 / 2}}$, -expressed in Rydberg- can be reasonably well predicted in the limit $\left|\mathcal{E}_{n^{\prime} S_{1 / 2}}\right|,\left|\mathcal{E}_{n^{\prime \prime} D_{3 / 2}}\right| \ll 1$. In this way, we are left with a single parameter which can be deduced from the empirical knowledge of 
another physical quantity involving the same matrix elements. We have in mind the quadrupolar amplitude $E_{2}^{h f}$ induced by the hyperfine interaction which is present in the cesium $6 S \rightarrow 7 S$ transition in the absence of a static electric field [2\&]. In order to show the relation between the quantities $A_{\|}-A_{\perp}$ and $E_{2}^{h f}$, we express them explicitly in terms of the matrix elements $M\left(n^{\prime}, n\right)$ given by:

$$
M\left(n^{\prime}, n\right)=\sum_{n^{\prime \prime}} \frac{\left\langle n^{\prime} S_{1 / 2}\left|H_{h f}\right| n^{\prime \prime} D_{3 / 2}\right\rangle\left\langle n^{\prime \prime} D_{3 / 2}\left|\rho^{2}\right| n S_{1 / 2}\right\rangle}{\mathcal{E}_{n^{\prime} S_{1 / 2}}-\mathcal{E}_{n^{\prime \prime} D_{3 / 2}}} .
$$

The basic formula used in our numerical evaluation of $A_{\|}-A_{\perp}$ can be cast in a very compact form:

$$
A_{\|}-A_{\perp}=-\frac{4 \lambda_{b}}{\Delta \mathcal{E}} \frac{2 M(6,6)}{M(7,6)+M(6,7)} a_{3}(7,6) R y
$$

where $\Delta \mathcal{E}$ is the energy of the $6 S \rightarrow 7 S$ transition and $a_{3} \propto E_{2}^{h f} / \mu_{B}$ is the empirical quadrupolar amplitude (see Eq. (A.2) for a precise definition). A second empirical input is used to determine the coupling constant $\lambda_{b}$ : this is the $S-D$ mixing coefficient which is obtained from the hyperfine frequency shifts measured by Weis et al. for Cs atoms trapped either in the (bcc) or the (hcp) phases [26] in the low magnetic field limit. The ratio involving the matrix elements $M\left(n^{\prime}, n\right)$ is evaluated in the appendix, using the approximation scheme sketched above. Its absolute value is found to lie close to unity. Let us quote now the final result given by our semi-empirical method[] described in the appendix: $\left|\frac{A_{\|}-A_{\perp}}{A_{\|}+A_{\perp}}\right|=1.07 \times 10^{-3}$. The uncertainty is believed not to exceed $20 \%$.

For observing the electroweak linear Stark shift discussed in the present paper, it is important to work with a uniaxial hexagonal crystal. Indeed, in a polycristalline phase, where the individual crystals are oriented totally at random, the average value of the pseudoscalar $\mathcal{P}$ taken over the isotropic distribution of $\hat{n}$ is expected to be suppressed and thus is the Stark shift computed in the previous section. Although trapping of cesium atoms has not yet been achieved in a monocrystalline hexagonal phase, the prospect does not look unfeasible [29] and a determination of the magnitude of the hyperfine anisotropy appears to be the first step to be achieved. Using $\left|\frac{A_{\|}-A_{\perp}}{A_{\|}+A_{\perp}}\right|=1.07 \times 10^{-3}$ and Eq. (34), we find that the effective P-odd T-even electric dipole moment of the trapped cesium atoms associated with the nuclear anapole moment reaches $2.96 \times 10^{-16}|e| a_{0}$. For comparison, it is interesting to note that this is about three times as large as the Cs EDM limit (Eq.20) to be measured on unperturbed Cs atoms for improving our present knowledge about a possible P-odd T-odd EDM of the electron.

\footnotetext{
${ }^{7}$ This method can be seen as a generalization of that used in sec.1.2 to evaluate the static dipole starting from the empirical knowledge of the transition dipole.
} 


\section{Breaking the free atom symmetry by application of a nonresonant radiation field}

In this last section we want just to mention another possibility for breaking the atomic Hamiltonian rotation symmetry by other means than static uniform electric and magnetic fields. We have in mind the application of a strong nonresonant radiation field which generates an anisotropic electron gyromagnetic ratio. In the presence of an external magnetic field $\vec{B}$ it has been shown [30] that the effect of the nonresonant radiation field can be described by the introduction of an effective magnetic field:

$$
\vec{B}^{\prime}=\left(g_{\perp} \vec{B}+\left(g_{\|}-g_{\perp}\right) \hat{n} \cdot \vec{B} \hat{n}\right) / \sqrt{\left(g_{\|}^{2}+g_{\perp}^{2}\right)},
$$

where $\hat{n}$ defines the direction of polarization of the radiation field, $g_{\perp}=g_{F}$ and $g_{\|}=$ $g_{F} J_{0}\left(\omega_{1} / \omega\right), J_{0}$ is the zero-order Bessel function, and $\omega_{1}$ is the Rabi angular frequency associated with the radiation field. The above formula suggests the existence of a uniaxial symmetry, but it is valid only within an atomic hyperfine multiplet. It is clear that the "dressing" by a nonresonant radiation field offers new possibilities for placing the atoms in a quadrupolar environment. However, it is important to bear in mind that at least two stringent requirements must be satisfied if one wants to detect an electroweak Stark shift in the ground state. First, the uniaxial perturbation has to mix the two hyperfine substates, otherwise the matrix element of $H_{p v}^{s t}$ cancels. Second, it is imperative to avoid a broadening of the transition lines for allowing precise frequency measurements. We are currently investigating how to achieve the proper conditions in a realistic way.

\section{Conclusion}

This paper investigates a way to get around the well known no-go theorem:

no linear Stark shift can be observed in a stationary atomic state unless $T$ reversal invariance is broken.

The perturbation of an atom by the nuclear spin-dependent parity-odd potential generated by the nuclear anople moment leads to a static electric dipole moment $d_{I} \vec{s} \wedge \vec{I}$, which clearly is T-even. However, if one considers an atom placed in arbitrarily oriented electric and magnetic uniform static fields $\vec{B}_{0}$ and $\vec{E}_{0}$, the quantum average $\vec{E}_{0} \cdot\langle\vec{s} \wedge \vec{I}\rangle$ is found to vanish. This can be understood by noting that $\vec{s} \wedge \vec{I} \cdot \vec{E}_{0}$ is odd under the quantum symmetry transformation $\Theta$ defined as the product of the time reflexion $T$ by a space rotation of $\pi$ about an axis normal to a plane parallel to

the fields $\vec{B}_{0}, \vec{E}_{0}$, while the atomic hamiltonian stays even. Our strategy to obtain a linear Stark shift is to break the $\Theta$ symmetry while keeping $\mathrm{T}$ invariance.

As a possible practical realization of such a situation, we have studied the case of ground state Cs atoms trapped in a uniaxial (hcp) phase of solid ${ }^{4} \mathrm{He}$, which has been 
recently the subject of detailed spectroscopic studies [26]. The required breaking of space rotation is provided by the uniaxal crystal field. As a result of the deformation of the atomic spatial wave function the hyperfine interaction acquires an anisotropic part, which plays an essential role in the determination of the size of the linear Stark shift. We have performed a numerical estimation of the hyperfine anisotropy, believed to be accurate to the $20 \%$ level, using a semi-empirical method. We use as an input the recent experimental measurement of the $E_{2}$ amplitude of the $6 S_{1 / 2} \rightarrow 7 S_{1 / 2}$ transition induced in cesium by the hyperfine interaction. We arrive in this way at a numerical evaluation of the linear Stark shift induced by the nuclear anapole moment: the expected effect is found to be about three times the experimental upper limit to be set on the T-odd Stark shift of free Cs atoms for improving the present limit on the electron EDM.

Besides the obvious remark that the T-even Stark shift studied here could be a possible source of systematic uncertainty in EDM experiments designed to reach unprecedented sensitivity [31, 21, 32, 33], we believe that there are strong physical motivations for measuring the Stark shift itself. First, it would lead to a direct measurement of the nuclear anapole moment in absence of any contribution coming from the dominant PV potential due to the weak nuclear charge. It would also provide an evidence for a truly static manifestation of the electroweak interaction, something which is still lacking. Second, this experiment would rely on the measurement of frequency shifts rather than transition amplitudes. While transition probabilities are difficult to measure very accurately, high precision measurements of frequency shifts have already been achieved.

\section{Acknowledgements}

We thank Ph. Jacquier for continuous interest in the subject of this work and his encouragements. We acknowledge many stimulating discussions with A. Weis and S. Kanorsky. We are grateful to M. Plimmer and J. Guéna for careful reading of the manuscript.

This work has been supported by INTAS (96-334). 


\section{APPENDIX: Semi-empirical calculation of the hyperfine anisotropy of Cs atoms trapped inside a ${ }^{4} \mathrm{He}$ hexagonal matrix}

In this appendix we present our evaluation of the hyperfine structure anisotropy $\frac{A_{\|}-A_{\perp}}{A_{\|}+A_{\perp}}$ resulting from the matrix induced bubble deformation of quadrupolar symmetry, a quantity frequently referred to in this paper.

\section{Two processes induced by hyperfine mixing}

Our approach is based on the fact that hyperfine mixing plays quite similar roles in two different processes. The first process concerns the Cs $6 S \rightarrow 7 S$ quadrupolar transition amplitude in zero electric field while the second process deals with the parameter $\frac{A_{\|}-A_{\perp}}{A_{\|}+A_{\perp}}$.

We start by rewriting the standard mixed $M_{1}-E_{2}$ transition operator in atomic units:

$$
T_{M_{1}+E_{2}}=(\vec{\epsilon} \wedge \vec{k}) \cdot \frac{\overrightarrow{\mathcal{M}}}{\mu_{B}}-i \frac{1}{2} \Delta \mathcal{E}(\vec{\rho} \cdot \vec{\epsilon})(\vec{\rho} \cdot \vec{k}),
$$

where $\vec{\rho}$ is the electron coordinate in Bohr radius unit and $\Delta \mathcal{E}$ is the transition energy expressed in Rydberg unit?. We are first going to study the perturbation effect on $T_{M_{1}+E_{2}}$ caused by the hyperfine interaction $H_{h f}$. This phenomenon has been observed experimentally in the forbidden $6 S_{1 / 2} \rightarrow 7 S_{1 / 2}$ transition. It provides a useful calibration amplitude in cesium parity violation experiments. To analyse the experimental results, it was found convenient, to introduce the effective transition operator $T_{h f}$ acting upon the tensor products of the electron spin and nuclear spin states:

$$
T_{h f}=i a_{2}\left(n^{\prime}, n\right)(\vec{s} \wedge \vec{I}) \cdot(\vec{\epsilon} \wedge \vec{k})+i a_{3}\left(n^{\prime}, n\right)((\vec{s} \cdot \vec{k})(\vec{I} \cdot \vec{\epsilon})+(\vec{s} \cdot \vec{\epsilon})(\vec{I} \cdot \vec{k}))
$$

The second physical process to be analysed in this section is not at first sight closely connected but happens to be described by the same formalism. This will allow us to establish a very useful connection between measurements coming from rather different experimental situations. Recently optical pumping has been observed with cesium atoms trapped inside an hexagonal matrix of solid helium [26]. Among the new effects to be expected, we have seen earlier in this paper that the existence of an anisotropic hyperfine structure opens the possiblity of observing a linear Stark shift induced by the nuclear anapole moment, an effect which cannot exist for an atom in a spherically symmetric environment. It is known that in the bubble enclosing the

\footnotetext{
${ }^{8}$ The phase difference, $\pi / 2$, between the two amplitudes expresses the fact that the magnetic moment $\overrightarrow{\mathcal{M}}$ and the quadrupole operator behave differently under time reflexion: the first is odd, while the second is even.
} 
cesium atom there is a small overlap between the cesium and the helium orbitals [23]. As a consequence, the axially symmetric crystal potential inside the bubble can be well approximated by a regular solution of the Laplace equation:

$$
H_{b}(\vec{n})=\lambda_{b}\left(\frac{e^{2}}{2 a_{0}}\right)\left((\vec{\rho} \cdot \vec{n})^{2}-\frac{1}{3} \rho^{2}\right)
$$

The perturbation of the hyperfine interaction by the bubble quadrupole potential $H_{b}(\vec{n})$ induces an anisotropic hyperfine structure for cesium $n S_{1 / 2}$ states. This is described by the effective Hamiltonian:

$$
H_{h f}^{\text {anis }}=\left(A_{\|}-A_{\perp}\right)\left((\vec{s} \cdot \vec{n})(\vec{I} \cdot \vec{n})-\frac{1}{3} \vec{s} \cdot \vec{I}\right) .
$$

We present now the basic formulas which allow the computation of the parameters relevant for the two physical problems in hand. They will be given in such a way as to exhibit their close anology. We have chosen to use the Dirac equation formalism. Besides the fact that formulas are more compact, it is well known that relativistic corrections play an important role in cesium hyperfine structure computation. Neglecting the contribution of the quadrupole nuclear moment of the Cs nucleus? the hyperfine hamiltonian is written as:

$$
\begin{gathered}
H_{h f}=\vec{I} \cdot \overrightarrow{\mathcal{A}} \\
\overrightarrow{\mathcal{A}}=C_{h f} \frac{\vec{\alpha} \wedge \vec{\rho}}{\rho^{3}}+\delta \overrightarrow{\mathcal{A}}^{(1)}\left(\vec{\rho}, \vec{\rho}^{\prime}\right)+\ldots
\end{gathered}
$$

The first term gives the hyperfine interaction of the valence electron treated as a Dirac particle; the second represents the non-local modification of the hyperfine interaction induced by the excitation of core electron-hole pairs to lowest order and the dots stand for higher order contributions ${ }^{\text {to }}$. It has been shown in reference [27 that the offdiagonal matrix element $\left\langle n^{\prime \prime} D_{3 / 2}\left|\frac{\vec{\alpha} \wedge \vec{\rho}}{\rho^{3}}\right| n S_{1 / 2}\right\rangle$ is strongly suppressed by an approximate selection rule which does not apply to the many-body non local operator $\delta \overrightarrow{\mathcal{A}}^{(1)}\left(\vec{\rho}, \vec{\rho}^{\prime}\right)$. An evaluation of the latter contribution led to a semi-quantative agreement with the experimental measurements of the ratio $a_{3}(7,6) / a_{2}(7,6)$, while the single particle result is too small by about two orders of magnitude.

\footnotetext{
${ }^{9}$ As shown in ref [28], the quadrupole contribution for ${ }^{133}$ Cs plays a negligible role in the effects discussed in this appendix.

${ }^{10}$ An explicit construction of $\delta \overrightarrow{\mathcal{A}}^{(1)}\left(\vec{\rho}, \vec{\rho}^{\prime}\right)$ together with a resummation of an infinite set of higher order terms, within the many body field theory formalism, is given in reference [34]. See also 35] for more advanced analysis.
} 
To obtain an estimate of the ratio $\left(A_{\|}-A_{\perp}\right) / a_{3}(7,6)$ it is convenient to introduce the cartesian tensor operator $T_{i_{1} i_{2} i_{3}}(E)$. This object appears naturally in the lowestorder pertubation expressions for the quantities of interest:

$$
T_{i_{1} i_{2} i_{3}}(E)=\mathcal{A}_{i_{1}} G_{3 / 2}^{+}(E)\left(\rho_{i_{2}} \rho_{i_{3}}-\frac{1}{3} \delta_{i_{2}, i_{3}} \rho^{2}\right)
$$

where the indices $i_{1}, i_{2}, i_{3}$ take any value between 1 and 3 . The scalar operator $G_{3 / 2}^{+}(E)$ is the atomic Green function operator restricted to the subspace of $D_{3 / 2}$ configurations (total atomic angular momentum $\mathrm{J}=3 / 2$ and positive parity). We now proceed to isolate in $T_{i_{1} i_{2} i_{3}}(E)$ the part transforming as a vector; this is the only part to survive after the operator is sandwiched between the projectors $P\left(n^{\prime} S_{1 / 2}\right)$ and $P\left(n S_{1 / 2}\right)$. This operation is achieved by a decomposition of $T_{i_{1} i_{2} i_{3}}(E)$ into a traceless tensor $\bar{T}_{i_{1} i_{2} i_{3}}(E)$ and a remainder [36]:

$$
\begin{aligned}
T_{i_{1} i_{2} i_{3}}(E)= & \bar{T}_{i_{1} i_{2} i_{3}}(E)+\frac{3}{10}\left(\delta_{i_{1}, i_{2}} T_{\alpha \alpha i_{3}}(E)+\delta_{i_{1}, i_{3}} T_{\alpha \alpha i_{2}}(E)\right) \\
& -\frac{2}{10} \delta_{i_{2}, i_{3}} T_{\alpha \alpha i_{1}}(E)
\end{aligned}
$$

where we have used the fact that $T_{\alpha \alpha i}=T_{\alpha i \alpha}$ and $T_{i \alpha \alpha}=0$. It is a simple matter to verify from the above equation that we have indeed $\bar{T}_{\alpha \alpha i_{3}}=\bar{T}_{\alpha i_{2} \alpha}=\bar{T}_{i_{1} \alpha \alpha}=0$. The fully symmetric part of the traceless tensor $\bar{T}_{i_{1} i_{2} i_{3}}^{S}(E)$ is easily identified with an octupole spherical tensor having seven independant components. By a simple counting argument, the left over term is seen to have five components; it is to be identified with the quadrupole tensor which appears in the full decomposition of $T_{i_{1} i_{2} i_{3}}(E)$ into irreducible representations of the rotation group $O(3)$. Let us have a look at the vector operator, $\vec{V}$, the components of which appear in the right hand side of Eq.(A.8):

$$
\vec{V}=\left(\overrightarrow{\mathcal{A}} G_{3 / 2}^{+}(E) \cdot \vec{\rho}\right) \vec{\rho}-\frac{1}{3} \overrightarrow{\mathcal{A}} G_{3 / 2}^{+}(E) \rho^{2} .
$$

The second term in the above expression does not contribute when it acts upon an $n S_{1 / 2}$ state so, we are led for our purpose to introduce the vector operator $\overrightarrow{\mathcal{T}}\left(n^{\prime}, n\right)$

$$
\begin{aligned}
\overrightarrow{\mathcal{T}}\left(n^{\prime}, n\right) & \left.=\frac{3}{10} P\left(n^{\prime} S_{1 / 2}\right)\left(\overrightarrow{\mathcal{A}} G_{3 / 2}^{+}\left(E_{i}\right) \cdot \vec{\rho}\right) \vec{\rho}+\left(h . c, E_{f} \rightarrow E_{i}\right)\right) P\left(n S_{1 / 2}\right) \\
& =\gamma\left(n^{\prime}, n\right) \vec{s}
\end{aligned}
$$

where $E_{f}$ and $E_{i}$ are respectively the binding energies of the $n^{\prime} S_{1 / 2}$ and $n S_{1 / 2}$ atomic states. The second line of the above equation follows directly from the Wigner-Eckart theorem applied to a vector operator. 
In order to calculate $a_{3}\left(n^{\prime}, n\right)$ we have to perform the contraction of $I_{i_{1}} \epsilon_{i_{2}} k_{i_{3}}$ with the tensor:

$$
F_{i_{1} i_{2} i_{3}}=P\left(n^{\prime} S_{1 / 2}\right)\left(T_{i_{1} i_{2} i_{3}}\left(E_{f}\right)+\left(\text { h.c., } E_{f} \rightarrow E_{i}\right)\right) P\left(n S_{1 / 2}\right) .
$$

Using Eq.(A目) and (A9), $F_{i_{1} i_{2} i_{3}}$ can be cast into the simple form :

$$
F_{i_{1} i_{2} i_{3}}=\gamma\left(n^{\prime}, n\right)\left(\delta_{i_{1}, i_{2}} s_{i_{3}}+\delta_{i_{1}, i_{3}} s_{i_{2}}-\frac{2}{3} \delta_{i_{2}, i_{3}} s_{i_{1}}\right) .
$$

The required index contraction with the tensor $I_{i_{1}} \epsilon_{i_{2}} k_{i_{3}}$ is now easily performed and one obtains directly $a_{3}\left(n^{\prime}, n\right)$, up to a prefactor whose value is found by identification with Eq.(A.]1):

$$
a_{3}\left(n^{\prime}, n\right)=-\frac{1}{2} \Delta \mathcal{E} \gamma\left(n^{\prime}, n\right) .
$$

To calculate the hyperfine anisotropy $A_{\|}-A_{\perp}$, we follow the same lines but this time the contraction involves the tensor $I_{i_{1}}\left(n_{i_{2}} n_{i_{3}}-\frac{1}{3} \delta_{i_{2}, i_{3}}\right)$, the prefactor is fixed by comparison with Eq.(A.3) and the exchange $i_{2} \leftrightarrow i_{3}$ leads to two identical contributions. Hence,

$$
\begin{aligned}
A_{\|}-A_{\perp} & =2 \lambda_{b}\left(\frac{e^{2}}{2 a_{0}}\right) \gamma(n, n) \\
& =-\left(\frac{e^{2}}{2 a_{0}}\right) \frac{4 \lambda_{b}}{\Delta \mathcal{E}} \frac{\gamma(n, n)}{\gamma\left(n^{\prime}, n\right)} a_{3}\left(n^{\prime}, n\right) .
\end{aligned}
$$

The expression (A.12) looks to us a good starting point for numerical evaluation of $A_{\|}-A_{\perp}$ : besides the fact that several sources of uncertainties in the evaluation of $\gamma\left(n^{\prime}, n\right)$ are eliminated in the ratio $\frac{\gamma(n, n)}{\gamma\left(n^{\prime}, n\right)}$, it lends itself to the use of empirical information. One may note, here, a certain similarity with Eq. (7) of sec 1.2 used for the evaluation of the permanent dipole, $d_{I}$.

\section{Numerical evaluation}

We proceed now to a numerical evaluation of $A_{\|}-A_{\perp}$ in three steps, starting from formula (A.12).

The numerical value of the $6 S_{1 / 2} \rightarrow 7 S_{1 / 2}$ quadrupole amplitude $a_{3}(7,6)$ is readily obtained from measurements [28, 2] of the ratio

$$
a_{3}(7,6) / a_{2}(7,6)=E_{2} / M_{1}^{h f}=(5.3 \pm 0.3) \times 10^{-2},
$$


combined with a precise theoretical evalution of the magnetic dipole amplitude $a_{2}(7,6)=-\frac{M_{1}^{h f}}{2 \mu_{B}}=-0.4047 \pm 4 \times 10^{-4}$. We obtain finally:

$$
a_{3}(7,6)=(2.14 \pm 0.12) \times 10^{-7}
$$

The second step is the numerical estimate of the ratio $\gamma(6,6) / \gamma(7,6)$. This is more delicate and requires an assumption which has been shown to work in similar situations. To begin with, we have addressed the question ${ }^{2}$ of the origin and size of the variations of the off-diagonal matrix elements $\left\langle n S_{1 / 2}\left|H_{h f}\right| n^{\prime \prime} D_{3 / 2}\right\rangle$ with the radial quantum numbers $n$ and $n^{\prime \prime}$. It is of interest to remind that a very precise answer to this question has been already obtained in the case of cesium single particle matrix elements $\left\langle n L_{J}\left|H_{h f}^{s p}\right| n^{\prime} L_{J}\right\rangle$ with $L=0$ or 1 and with $n$ and $n^{\prime}$ referring to the radial quantum numbers of any pair of valence states. For simplicity, we are going to express the answer within a non-relativistic formalism, but it should be borne in mind that all of what is said holds true within a relativistic framework. It is convenient to introduce the notion of overline matrix elements such as those computed with radial wave functions $\overline{R_{n l j}}(\rho)$ which have a starting coefficient at the origin equal to unity instead of a unit norm [3]. More explicitly we can write:

$$
\overline{\left\langle n L_{J}\left|H_{h f}^{s p}\right| n^{\prime} L_{J}\right\rangle}=\frac{\left\langle n L_{J}\left|H_{h f}^{s p}\right| n^{\prime} L_{J}\right\rangle}{A_{n l_{j}} A_{n^{\prime} l_{j}}},
$$

where $A_{n l_{j}}=\lim _{\rho \rightarrow 0} \rho^{-l} R_{n l j}(\rho)$ is the starting coefficient of the space normalized wave function. (In the relativistic case the above condition is replaced by energy independent boundary conditions imposed on the Dirac radial wave functions at the nuclear radius). It was found in references [27, 37] that the overlined matrix elements are independent of the valence orbital radial quantum numbers $n$ and $n^{\prime}$ to better than $10^{-4}$ for $S_{1 / 2}$ states and better than $10^{-3}$ for $P_{1 / 2}$ states. This result is understood by noting that, in the domain of the $\rho$ values relevant for the evalutation of the matrix elements of $\frac{\vec{\alpha} \wedge \vec{\rho}}{\rho^{3}}$ for $S_{1 / 2}$ and $P_{1 / 2}$ states, the potential energy is larger than valence binding energies by more than three orders of magnitude. This implies that, in this domain, the overlined radial wave functions have no dependence upon the binding energy or equivalently upon the radial quantum numbers of the valence orbitals.

\footnotetext{
${ }^{11}$ The theoretical method used to get $M_{1}^{h f}$ is based upon the factorization rule: $\left\langle 6 S\left|H_{h f}\right| 7 S\right\rangle=$ $\sqrt{\left\langle 6 S\left|H_{h f}\right| 6 S\right\rangle\left\langle 7 S\left|H_{h f}\right| 7 S\right\rangle}$. This rule was first established with an accuracy of a few parts in $10^{3}$ in ref. [27]. It has been confirmed by a direct many-body relativistic computation [38] of $\left\langle 6 S\left|H_{h f}\right| 7 S\right\rangle$, accurate to the $1 \%$ level. More recently the validity of the rule has been pushed to the level of a fraction of $10^{-3}$ [39].

${ }^{12}$ Arguments similar to those given below and in references [27, 37] are developed in [39].

13 The wave function $\overline{R_{n l j}}(\rho)$ is known to be an analytic function of the energy. This property is the starting point of the quantum defect theory.
} 
The above argument has to be reconsidered for the lowest order many body correction involving the matrix element of the non local operator: $\delta \overrightarrow{\mathcal{A}}^{(1)}\left(\vec{\rho}, \vec{\rho}^{\prime}\right)$. The relevant domain of $\rho$ values is now determined by the "radii" of the core outer orbitals involved in the computation, which in the case of $S_{1 / 2}$ and $P_{1 / 2}$ matrix elements are $5 s, 5 p$, while in the case of the off-diagonal matrix element $\left\langle n S_{1 / 2}\left|\delta \overrightarrow{\mathcal{A}}^{(1)}\right| n^{\prime} D_{3 / 2}\right\rangle$ only $5 p$ is relevant. We measure the variation of the overlined matrix elements $\overline{\left\langle n L_{J}\left|H_{h f}^{m b}\right| n^{\prime} L_{J^{\prime}}^{\prime}\right\rangle}$ with the valence state binding energies $\mathcal{E}_{n L_{J}}$ by the parameters $\delta_{L_{J}}$ defined as their logarithmic derivative with respect to $\mathcal{E}_{n L_{J}}$, (here $H_{h f}^{m b}$ stands for the many-body modification to the hyperfine interaction). From results of references [27, 37], we can infer the relative variation of $\overline{\left\langle n L_{1 / 2}\left|\delta \overrightarrow{\mathcal{A}}^{(1)}\right| n^{\prime} L_{1 / 2}\right\rangle}$ for $L=0,1$ and we arrive to the values $\delta_{S_{1 / 2}}^{(1)}=-0.12$ and $\delta_{P_{1 / 2}}^{(1)}=-0.30$. The fact that $-\delta_{P_{1 / 2}}^{(1)}$ is about three times larger than $-\delta_{S_{1 / 2}}^{(1)}$ is coming from the fact that $P$ state binding energies have to be compared with the potential energy minus the centrifugal energy. Let us, now, consider the more difficult case of the S-D off-diagonal matrix elements $\overline{\left\langle n^{\prime} S_{1 / 2}|\delta \overrightarrow{\mathcal{A}}(1)| n^{\prime \prime} D_{3 / 2}\right\rangle}$. The corresponding parameter $\delta_{S_{1 / 2}}^{(1)}$ is expected to be somewhat larger in absolute value, due to the fact that the relevant $5 p$ orbital is less tightly bound than the $5 \mathrm{~s}$ orbital which gives the dominant contribution to the $S_{1 / 2}$ diagonal matrix element. The relative variation versus the $D_{3 / 2}$ energy is expected to be on the order of few units, since the centrigugal barrier is three times higher than in the case of $P$ states. This expectation is borne out by a preliminary estimate which gives $\delta_{D_{3 / 2}}^{(1)} \sim-3$.

We proceed now to a numerical evaluation of the ratio $r_{\text {anis }}=\gamma(6,6) / \gamma(7,6)$, leaving, for the moment, $\delta_{S_{1 / 2}}$ and $\delta_{D_{3 / 2}}$ as free parameters. As an intermediate step, we compute the quantities $M\left(n^{\prime}, n\right)$, written as sums over the intermediate $n^{\prime \prime} D_{3 / 2}$ states :

$$
M\left(n^{\prime}, n\right)=\sum_{n^{\prime \prime}} \frac{\left\langle n^{\prime} S_{1 / 2}\left|H_{h f}\right| n^{\prime \prime} D_{3 / 2}\right\rangle\left\langle n^{\prime \prime} D_{3 / 2}\left|\rho^{2}\right| n S_{1 / 2}\right\rangle}{\mathcal{E}_{n^{\prime} S_{1 / 2}}-\mathcal{E}_{n^{\prime \prime} D_{3 / 2}}} .
$$

The ratio $r_{\text {anis }}$ is given in terms of $M\left(n^{\prime}, n\right)$ by the following formula :

$$
r_{\text {anis }}=\gamma(6,6) / \gamma(7,6)=\frac{2 M(6,6)}{M(7,6)+M(6,7)} .
$$

An explicit numerical computation of $r_{\text {anis }}$ has been performed according to the following procedure. First, any binding energy independent factor appearing in $M\left(n^{\prime}, n\right)$ is dropped since it disappears in the ratio. This is indicated below by the symbol $\propto$. The sum $\sum_{n^{\prime \prime}}$ appearing in $M\left(n^{\prime}, n\right)$ is limited to $5 \leq n^{\prime \prime} \leq 8$. The set of the quadrupole matrix element $\left\langle n^{\prime \prime} D_{3 / 2}\left|\rho^{2}\right| n S_{1 / 2}\right\rangle$ were obtained by a relativistic version of the Norcross model. In order to test the sensitivity of the result to quadrupole amplitudes, we have also used a set calculated by an extension of the Bates-Damgaard method. The energy denominators are taken from experiment. The hyperfine matrix 
elements $\left\langle n^{\prime} S_{1 / 2}\left|H_{h f}\right| n^{\prime \prime} D_{3 / 2}\right\rangle$, to second order in the binding energies are, given by the following formulas:

$$
\begin{aligned}
\left\langle n^{\prime} S_{1 / 2}\left|H_{h f}\right| n^{\prime \prime} D_{3 / 2}\right\rangle \propto & A_{n^{\prime \prime} D_{3 / 2}} A_{n^{\prime} S_{1 / 2}} \times \\
& \left(1+\delta_{S_{1 / 2}} \mathcal{E}_{n^{\prime} S_{1 / 2}}+\delta_{D_{3 / 2}} \mathcal{E}_{n^{\prime \prime} D_{3 / 2}}\right), \\
A_{n L_{J}} \propto & \left(-\mathcal{E}_{n L_{J}}\right)^{\frac{3}{4}} .
\end{aligned}
$$

In formula (17), we have dropped, according to the above prescription, the zero energy limit of the overlined matrix element $\overline{\left\langle n^{\prime} S_{1 / 2}\left|H_{h f}\right| n^{\prime \prime} D_{3 / 2}\right\rangle}$. Equation (A.18) follows from a result obtained in [16], where the Fermi-Segré formula was extended to arbitrary orbital angular momentum states. For simplicity we have ignored for simplicity a factor involving the derivative of the quantum defects, which in the present context would introduce few percent corrections.

We now have all the elements needed to calculate the sought after ratio:

$$
r_{\text {anis }}=\frac{\gamma(6,6)}{\gamma(7,6)}=-0.8173-0.0255 \delta_{D_{3 / 2}}+0.1456 \delta_{S_{1 / 2}}
$$

The negative sign of $r_{\text {anis }}$ can be traced back to the fact that the $7 S_{1 / 2}$ level lies just in between $5 D_{3 / 2}$ and $6 D_{3 / 2}$ levels. If we adopt the rough estimate given above : a few tens of $\%$ for $-\delta_{S_{1 / 2}}$ and a few units for $-\delta_{D_{3 / 2}}$, the first-order energy correction remains well below the $10 \%$ level, due in part to a cancellation between the two correcting terms. To reduce the absolute value of $r_{\text {anis }}$ by more than $10 \%$ would require unrealistic values of $-\delta_{D_{3 / 2}}$ so we believe the estimate of $r_{\text {anis }}=-0.82 \pm 0.10$ to be reasonably safe ${ }^{\text {m. }}$.

The final step in our evaluation of the hfs anisotropy is devoted to the empirical determination of the coupling constant $\lambda_{b}$ appearing in front of the crystal electronic potential. As experimental input we are going to use the hyperfine energy shift which is observed for trapped cesium atoms, when one passes from the cubic to the hexagonal phase. This shift is attributed to the effect of the anisotropic bubble potential $H_{b}(\vec{n})$. We shall ignore, for the moment, the possible contribution of the anisotropic hyperfine interaction $H_{h f}^{a n i s}$ and assume that the shift is essentially due to the renormalization of the $6 S_{1 / 2}$ component of the atomic wave function by the admixtures $\alpha_{n D_{3 / 2}}$ of the $n D_{3 / 2}$ states. The corresponding variation of the hyperfine splitting $\delta W$ is then given by :

$$
\frac{\delta W}{W}=-\sum_{n, J}\left|\alpha_{n D_{J}}\right|^{2}=-\lambda_{b}^{2} \mathcal{J}_{S D},
$$

\footnotetext{
14 The validity of the procedure leading to this estimate has been checked, to the $10 \%$ level, upon a significative subset of the many-body Feynman diagrams contributing to $\gamma\left(n^{\prime}, n\right)$.
} 
where we have isolated $\lambda_{b}^{2}$ by introducing the purely atomic quantity $\mathcal{J}_{S D}$. Let us write down the explicit expression of $\mathcal{J}_{S D}$, neglecting spin-orbit coupling and assuming that $\vec{n}$ lies along the quantization axis:

$$
\mathcal{J}_{S D}=\sum_{n}\left|\left\langle 6 S\left|\left(\cos ^{2} \theta-1 / 3\right) \rho^{2}\right| n D\right\rangle /\left(\mathcal{E}_{6 S}-\mathcal{E}_{n D}\right)\right|^{2}
$$

We limit the sum to $n$ values ranging from 5 to 8 . With the same radial quadrupole matrix elements as before, we obtain the numerical value: $\mathcal{J}_{S D}=9512$. Using the empirical number given in ref [26], $\sqrt{-\delta W / W}=0.035$, we arrive at the following absolute value of the coupling constant $\lambda_{b}$ (in Ry):

$$
\left|\lambda_{b}\right|=\sqrt{\frac{-\delta W}{\mathcal{J}_{S D} W}}=0.000359 .
$$

It should be pointed out that if the crystal axis $\vec{n}$ is not aligned along the quantization axis, one obtains values of $\mathcal{J}_{S D}$ smaller than the one quoted above, so the value of $\left|\lambda_{b}\right|$ should be considered, strictly speaking, as a lower bound. At last, we have in hand all the ingredients needed to perform a numerical evaluation of $\left|A_{\|}-A_{\perp}\right|$ from the formula (A.12) since $\Delta \mathcal{E}=0.169$ is taken directly from experiment:

$$
\begin{aligned}
\left|A_{\|}-A_{\perp}\right| & =\frac{4\left|\lambda_{b}\right|}{\Delta \mathcal{E}} \frac{|\gamma(6,6)|}{|\gamma(7,6)|}\left|a_{3}(7,6)\right| \operatorname{Rydberg}(\mathrm{MHz})=4.9 \mathrm{MHz} \\
& =1.07 \times 10^{-3} \times\left(\left|A_{\|}+A_{\perp}\right|\right) .
\end{aligned}
$$

As a final topic, we should discuss the effect of the anisotropic hyperfine interaction itself on the the empirical splitting $\delta W$, since this could modify the value of $\left|\lambda_{b}\right|$ and so play a role in the assessment of the uncertainty affecting the result given by Eq. (A.24). Due to this effect, the constant $\lambda_{b}$ is no longer given by Eq. (A.22) but rather by a second order equation where the linear term is associated with the anisotropic hyperfine interaction. It is convenient to introduce the variable $x=\lambda_{b} / \lambda_{b}^{0}$ with $\lambda_{b}^{0}=\sqrt{-\delta W /\left(\mathcal{J}_{S D} W\right)}$. The equation giving $\lambda_{b}$ takes then the simple form: $x^{2}-2 b x-1=0$, where the coefficient $b$ is given by the following formula:

$$
b=\frac{\left(A_{\|}-A_{\perp}\right)^{(0)}}{A_{\|}+A_{\perp}} \frac{W}{\delta W} \frac{\Delta_{F}\left\langle s_{z} I_{z}-\frac{1}{3} \vec{s} \cdot \vec{I}\right\rangle}{2 I+1} .
$$

The superscript ${ }^{(0)}$ indicates that the hf anisotropy is given, up to a well defined sign, by Eq. (A.24). The symbol $\Delta_{F}$ means that one should take the difference between the two hyperfine states of the quantum average over which it is applied. To obtain an over-estimate of $b$ we have assumed that optical pumping works at its maximum 
efficiency so that the microwave transition takes place between the hyperfine levels $(4,4)$ and $(3,3)$. In this case we obtain $b=0.20$ and the two possible solutions for $\lambda_{b}$ are :

$$
\lambda_{b}^{( \pm)}= \pm 3.610^{-4}(1 \pm 0.2) .
$$

The actual experimental situation is expected to lie far from the extreme case considered here, so the difference between the two absolute values is certainly smaller than the upper limit given by the above calculation.

In conclusion, including all sources of uncertainties, we consider our evaluation of Eq.(A.24),$\left|\frac{A_{\|}-A_{\perp}}{A_{\|}+A_{\perp}}\right|=1.07 \times 10^{-3}$, as reliable within uncertainty limits of about $20 \%$. However, if, during hyperfine shift measurements $\vec{n}$ is not aligned along the

quantization axis, the central value of $\lambda_{b}$, and therefore that of $\frac{A_{\|}-A_{\perp}}{A_{\|}+A_{\perp}}$, may be pushed upwards.

\section{References}

[1] M. A. Bouchiat and C. Bouchiat, Rep. Prog. Phys. 60, 1351 (1997).

[2] S. C. Bennett and C. E. Wieman, Phys. Rev. Lett. 82, 2484 (1999).

[3] M. A. Bouchiat, in Electroweak Interactions and Unified Theories, (Proceedings of the $X X X V^{\text {th }}$ Rencontres de Moriond, March 11-18 2000 Tran Than Van ed., éditions Frontière).

[4] P. Fayet and G. Altarelli, inParity Violation in Atoms and Electron Scattering, B. Frois and M. A. Bouchiat eds. (World Scientific, 1999).

[5] M. J. Ramsey-Musolf,Phys. Rev. C 60, 015501 (1999).

[6] M. A. Bouchiat, J. Guéna, L. Hunter and L. Pottier, Phys. Lett. B 117, 358 (1982); Phys. Lett. B 134, 463 (1984).

[7] M. A. Bouchiat, J. Guéna, L. Hunter and L. Pottier, J. Phys. (France) 47, 1709 (1986).

[8] S. L. Gilbert, M. C. Noecker, R. N. Watts, and C. E. Wieman, Phys. Rev. Lett. 55, 2680 (1985).

[9] M. C. Noecker, B. P. Masterson, and C. E. Wieman, Phys. Rev. Lett. 61, 310 (1988).

[10] C. S. Wood et al, Science, 275, 1759 (1997). 
[11] V. V. Flambaum and I. B. Khriplovich, Sov. Phys. JETP 52, 835 (1980).

[12] Ya. B. Zel'dovich, Sov. Phys. JETP, 6, 1184 (1957).

[13] C. Bouchiat and C. A. Piketty, Z. Phys. C 49, 49 (1991).

[14] C. Bouchiat, in Parity Violation in Atoms and Electron Scattering, B. Frois and M. A. Bouchiat eds. (World Scientific, 1999) p. 138.

[15] V. V. Flambaum et al., Phys. Lett. B 146, 367 (1984).

[16] M. A. Bouchiat and C. Bouchiat, J. Phys. (France) 36, 899 (1974).

[17] D. Budker, in Proceedings of the Fifth International Symposium of the WEIN Conference (Santa Fe, June 98), P. Herczeg, C. M. Hoffman, H. V. KlapdorKleingrothaus, eds., (World Scientific, 1999), p. 418-441; and private communication.

[18] A. L. Barra, J. B. Robert, and L. Wiesenfeld, Phys. Lett. A 115, 443 (1986) and Europhys. Lett. 5, 217 (1988).

[19] P. G. H. Sandars, Physica Scripta 36, 904 (1987).

[20] S. A. Blundell, W. R. Johnson and J. Sapirstein, Phys. Rev. D 45, 1602 (1992).

[21] E. D. Commins, S. B. Ross, D. DeMille, and B. C. Regan, Phys. Rev. A 50, 2960 (1994).

[22] W. R. Johnson, D. S. Guo, M. Idrees, and J. Sapirstein, Phys. Rev. A 34, 1043 (1986); A. C. Hartley, E. Lindroth, and A. M. Martensson-Pendrill, J. Phys. B 23, 3417 (1990).

[23] S. I. Kanorsky, and A. Weis, in Advances in Atomic, Molecular and Optical Physics, vol38, B. Bederson and H. Walther eds., (Academia Press, San Diego, 1998), p. 87, and references therein.

[24] Ch. Daussy, T. Marrel, A. Amy-Klein, C. T. Nguyen, Ch. J. Bordé, and Ch. Chardonnet, et al., Phys. Rev. Lett. 83, 1554 (1999).

[25] M. Arndt, S. I. Kanorsky, A. Weis, and T. W. Hänsch, Z. Phys. 98, 377 (1995).

[26] S. Kanorsky, S. Lang, T. Eichler, K. Winkler and A. Weis, Phys. Rev. Lett. 81, 401 (1998).

[27] C. Bouchiat and C. A. Piketty, J. Phys. France 49, 1851 (1988). 
[28] M. A. Bouchiat and J. Guéna, J. Phys. France 49, 2037 (1988).

[29] S. Balibar, and A. Weis, private communications.

[30] C. Landré, C. Cohen-Tannoudji, J. Dupont-Roc and S. Haroche, J. Physique France 31, 971 (1970).

[31] L. R. Hunter, Science 25273 (1991).

[32] E. D. Hinds and B. Sauer, Physics World April 1997, p. 37.

[33] M. V. Romalis, W. C. Griffith, and E. N. Fortson, arXiv:hep-ex/0012001 1 Dec 2000.

[34] C. Bouchiat, in Atomic Physics 7, D. Kleppner and F. M. Pipkin eds. (Proceedings of the Seventh International Conference on Atomic Physics, Plenum Press, New York, 1981), p. 83-119.

[35] V. A. Dzuba, V. V. Flambaum, A. Ya Kraftmakher, and O. P. Sushkov, Phys. Lett. A 142, 373 (1989).

[36] Morton Hamermesh, Group Theory, (Addison-Wesley series in Physics, Pergamon Press: London-Paris, 1962), p. 393.

[37] C. Bouchiat and C. A. Piketty, Europhys. Lett. 2, 511 (1986).

[38] A. Derevianko, M. S. Safronova and W. R. Johnson, Phys. Rev. A 60, R1741 (1999).

[39] V. A. Dzuba and V. V. Flambaum, Phys. Rev. A 62, 052101 (2000). 